\title{
Herpes Simplex Virus Ophthalmic Disease Induced Using Two Different Methods of Mice Inoculation
}

Sílvia Regina Ferreira Gonçalves Pereira, Maria Angélica A. M. Guimarães, Leonardo V. Neto, Daniel Segenreich, Rafael Brandão Varella, Vera Lucia Antunes Chagas and Fernando Portela Câmara

\begin{abstract}
Laboratory of Viral Pathogenesis and Diagnosis, Department of Virology, Professor Paulo de Góes Institute of Microbiology, Center for Health Sciences, Federal University of Rio de Janeiro; Laboratory of Virology, Clinical Pathology Service, Clementino Fraga Filho Academic Hospital; Department of Pathology, Service of Pathological Anatomy; Martagão Gesteira Institute of Child Care and Pediatrics; Laboratory of Epidemiology of Viral Diseases, Federal University of Rio de Janeiro, Rio de Janeiro, RJ, Brazil
\end{abstract}

Two different procedures for inoculation of HSV on corneas of BALB/c mice were evaluated. The first was by the use of HSV suspensions directly on the corneas and the other was after corneal scarification. Animals by this later method presented greater morbidity and mortality than those of first group, suggesting that inoculation of HSV without scarification of the cornea should be the method of choice for the study of HSV ophthalmic infection. This model showed also be an efficient experimental system to testing antiviral drugs.

Key Words: Herpes simplex virus, ophthalmic disease, non-traumatic and traumatic inoculation, murine model.

Herpes simplex virus is a cosmopolite virus with significative prevalence of ophthalmic infection in both developed and underdeveloped countries [1]. This infection may be asymptomatic or be followed by conjunctivitis, blepharitis and keratitis that may result in corneal scarring and neovascularization with corresponding reduction of the vision $[2,3]$. It is the most prevalent cause of corneal opacification, blindness

Received on 21 March 2001; revised 27 May 2001.

Address for correspondence: Dr. Sílvia Regina Ferreira Gonçalves Pereira. Rua Barão de Mesquita, 850, Bloco E, Apto. 1212, Tijuca, Zip code: 20540-004, Rio de Janeiro, RJ, Brazil. Phone: 55 (02121) 208-5190, 55 (02121) 571-9589

E-mail: silviareginafgp@ig.com.br

Support: This study was supported by grants from CAPES, CNPq, FUJB, FAPERJ, and FINEP.

The Brazilian Journal of Infectious Diseases 2001;5(4):183-191 (C) 2001 by The Brazilian Journal of Infectious Diseases and Contexto Publishing. All rights reserved.

$1413-8670$ and secondary glaucoma in humans worldwide [1]. Approximately $90 \%$ of HSV ophthalmic infection are caused by the virus type 1 (HSV-1) and the remainder by the type 2 (HSV-2) [4-6]. Despite advances in many areas of virology we are still unable to prevent HSV infection and recurrence.

Different animal species have been used for the study of HSV pathogenesis and for the testing of new antiviral drugs. Rabbits have been the best animal selected to HSV ophthalmic infection using inoculation without previous corneal scarification. The use of mice to study HSV ophthalmic infection has been already described using the corneal scarification method to inoculate virus [7-14]. However, this form of inoculation produces frequently corneal scarring and it is unrealistic regarding the natural way of HSV ophthalmic infection in humans. Mice are less expensive than rabbits and more practical for laboratory use, so, its use may be advantageous in large scale experiments. 
In order to evaluate the clinical consequences of the two forms of HSV inoculation (with and without corneal scarification) we have studied a group of BALB/c mice which had no corneal trauma (group A animals) and another group that was submitted to corneal scarification before inoculation (group B animals). Morbidity and mortality assessment, virus isolation in cell culture, HSV antigen detection by indirect immunofluorescence method and histopathological aspects of the infected eye were used to evaluate the two methods.

\section{Materials and Methods}

\section{Animal inoculation}

62 male inbred 3-week-old BALB/c mice were used in the experiments. The mice were anesthetized before inoculation by ether (Merck) inhalation and examined with the aid of a slit-lamp biomicroscope (SL 5 model; Kowa Co., Ltd., Nagoya, Japan) to exclude any animals with corneal trauma. The first group of animals (20 animals of group A) was inoculated without scarification of the cornea. The following group (20 animals of group B) had its corneas scarificated before inoculation with the aid of a sterile 25-gauge needle, three times vertically and three times horizontally. Both groups of animals were inoculated with $10 \mathrm{~mL}$ of HSV1 strain (adapted to Vero cells) containing 106.5 infectious particles $\left(100 \mathrm{CCID}_{50} / \mathrm{mL}\right)$. Control mice were inoculated with non-infected material either by a non-traumatic ( 5 mice of group $C$ ) or the scarification procedure (5 mice of group D) using a $10 \mathrm{~mL}$ suspension of uninfected Vero cells (mock inoculum). An additional 12 mice ( 3 mice per group A-D) were studied using histological techniques (see below). All mice were handled according to the National Institute of Health (NIH) guidelines and the Association for Research in Vision and Ophthalmology (ARVO).

\section{Clinical evaluation of the infected and control animals}

Mice eyes were examined daily with a slit-lamp biomicroscope from day 1 to 14 after inoculation.
Before clinical examination the animals were anesthetized by ether inhalation and the signs of conjunctivitis, epithelial keratitis, blepharitis, stromal keratitis and neovascularization were recorded. The percentages of animals showing each of the observed symptoms were determined.

\section{Virus isolation in Vero cell culture}

To assess the laboratory confirmation of HSV infection, mouse eye swabs were taken daily from day 1 until day 10. Cotton swabs were transferred to a transport medium for inoculation in Vero (African green monkey kidney) cell monolayers cultivated in $13 \times 100 \mathrm{~mm}$ tubes.

Indirect immunofluorescence for the detection of HSV antigen

In order to detect virus replication in the eyes of the infected animals, corneal imprints were made onto glass slides. Tissue imprints were airdried and fixed in cold acetone 100\% (Merck). A rabbit anti-HSV antibody (DAKO) and a goat anti-rabbit antibody conjugated to fluorescein (DAKO, FITC) were used for the indirect immunofluorescence (IF) method.

\section{Histological examination of the eyes}

For the histological study, another 12 male inbred 3-week-old BALB/c mice were used (3 mice of each group A, B, C, and D) and inoculated as before. Mouse corneas of groups B and D were scarified as described before, while those of groups $\mathrm{A}$ and $\mathrm{C}$ were not scarificated. Animals were also anesthetized by ether inhalation and were examined under a slit-lamp biomicroscope, before inoculation. For the histological study they were sacrificed by ether overdose at the $2^{\text {nd }}, 5^{\text {th }}, 10^{\text {th }}$ and $14^{\text {th }}$ day a.i., their eyes were enucleated and immediately fixed in a $10 \%$ neutral buffered formalin. A 5-mm paraffinembedded sections were prepared, and stained with hematoxylin and eosin (H\&E) and mounted with Permount for light microscopic examination. The animals used in the histological studies were not included in the clinical studies. 


\section{Statistical analysis}

The results were analysed by ANOVA test using the software Primer.

\section{Results}

This study compares two groups of mice (GA and GB) inoculated by two different methods for compare the clinical and virological results (Figures 1,2 and 3) of the experimental ophthalmic infection produced by our procedures. The results of HSV isolation and antigen detection from corneal imprints of the infected mice is showed in the Figure 4, and the histopathological aspects of the infected eyes in the Figure 5.

During the daily clinical observation of the animals were noticed that conjunctivitis was the first symptom observed in both groups of infected animals, being earlier in the GB $(\mathrm{P}<0.01)$. It took 6 days and 3 days to reach $100 \%$ of the GA and GB animals, respectively, suggesting that corneal scarification might help dissemination (Figures 2 and 3). GB animals showed signs of epithelial keratitis after the first day a.i. reaching $100 \%$ of the animals on the following day, in GA animals it was observed on day 2 a.i., reaching $100 \%$ of the animals on the day 8 a.i. $(\mathrm{P}<0.05)$. Blepharitis was observed between 2 and 3 days a.i. in GB animals, and between the day 3 and 4 a.i. in GA animals. $100 \%$ of GB animals had blepharitis at day 5 a.i., while GA animals had this peak of symptom only on day 10 a.i. $(\mathrm{P}<0.01)$. The analysis of the 2 infected groups revealed that GB presented a greater number of animals with these symptoms in comparison with GA animals $(\mathrm{P}<0.05)$.

GB animals presented stromal keratitis and neovascularization after day 3 a.i., reaching a maximum of $65 \%$ and $60 \%$, respectively, on day 5 a.i.. GA animals manifested stromal keratitis and neovacularization only between the days 6 and 7 a.i., and $40 \%$ of the animals had these observed symptoms on day 10 a.i. $(\mathrm{P}<0.05)$. $50 \%$ of GB animals died by day 16 after inoculation, compared to $10 \%$ in GA group.

All of the infected animals were positive to HSV isolation in Vero cell culture confirmed by the HSV antigen detection by IF after corneal imprint using specific HSV antibody (Figure 4), and the histopathological study of their HSV infected eyes showed lesions compatible with HSV ophthalmic infection (Figure 5).

\section{Discussion}

Our findings of blepharitis lasting more than 14 days a.i. in animals of GA and GB are in accordance with the studies of Maggs, et al. [15]. According to these authors, the presence of HSV DNA in the epithelial cells of conjunctiva, hair follicles, and epidermal cells of the eyelid skin suggests that these events could be responsible for the chronic inflammatory lesions present in HSV ophthalmic infection.

It is possible that corneal scarification intensified immunological phenomena in HSV infection and contributed to the difference observed in the 2 infected groups regarding the number of animals that evolved to stromal keratitis and neovascularization. Probably virus replication in the presence of a great number of neutrophils, due to trauma of the cornea accelerated the inflammatory response. Stromal keratitis and neovacularization have an immune-mediated pathogenesis that involves T-cells and neutrophils [8] that are the most prominent cell types to migrate, initially, into HSV infected murine cornea [16].

Our results suggest that the inoculation of $\mathrm{HSV}$ after corneal scarification increases both morbidity and mortality. Virus isolation and histopathologic changes were similar in the 2 groups There were sufficient clinical signs and symptoms and lower mortality in the nonscarified group of animals to study the pathogenesis of the disease. Inoculation without scarification of the cornea should be the method of choice for the study of latency and antiviral drug efficiency.

\section{Acknowledgements}

We are grateful to José Carlos Dias Cunha, Nádia Maria dos Santos Marques and Emília Rosária Carvalho dos Santos (Laboratory of Pathology, Service 
Figure 1. Clinical aspects of the herpetic ophthalmic infection in mouse eyes. A, Group A animal eye showing intense conjunctival hyperemia with purulent discharge, almost $50 \%$ of the cornea involved with corneal opacification, edema eyelids and vesicles with moderate crusting; these findings configuring, respectively, conjunctivitis, epithelial keratitis and blepharitis (6 days a.i.). B, Group B animal eye showing severe disease, lid ulceration with hair loss, blepharitis, conjunctivitis, epithelial keratitis, stromal keratitis and central corneal geographic ulceration (10 days a.i.).
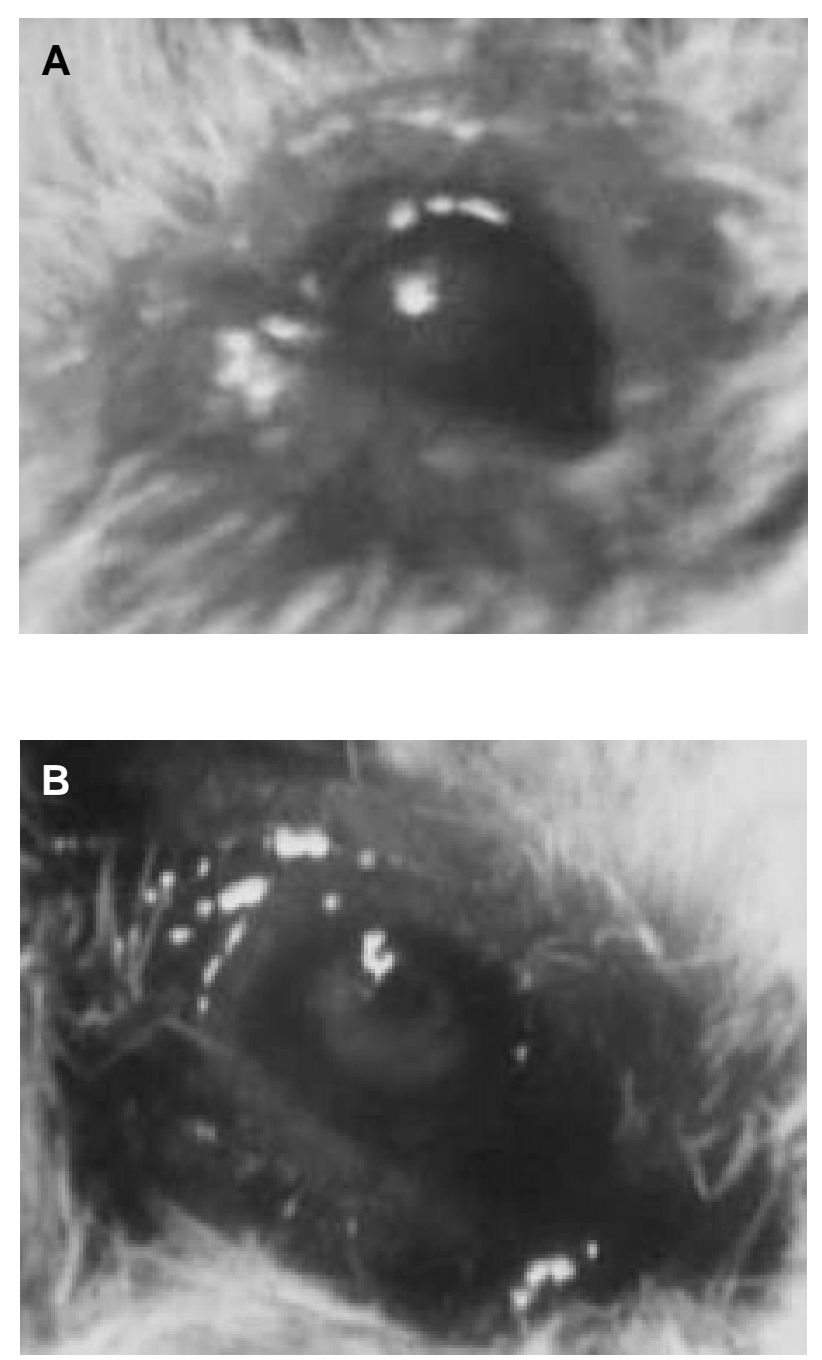
Figure 2. Graphic representation of group A mice (suspension only) with HSV ophthalmic infection. The data shows that conjunctivitis $(O)$ was the first observed symptom and took 6 days to reach $100 \%$ of the group A animals. Epithelial keratitis ( $\bullet$ ) was observed since the $2^{\text {nd }}$ day a.i. reaching $100 \%$ of the animals at the $8^{\text {th }}$ day a.i. Blepharitis $(\triangle)$ was observed between the $3^{\text {rd }}$ and $4^{\text {th }}$ days a.i. reaching $100 \%$ of the animals at the $10^{\text {th }}$ day a.i.. Stromal keratitis $(\Delta)$ and neovascularization $(\square)$ were observed between the $6^{\text {th }}$ and $7^{\text {th }}$ day a.i., and no more than $40 \%$ of group A animals had these observed symptoms at the $10^{\text {th }}$ day a.i.

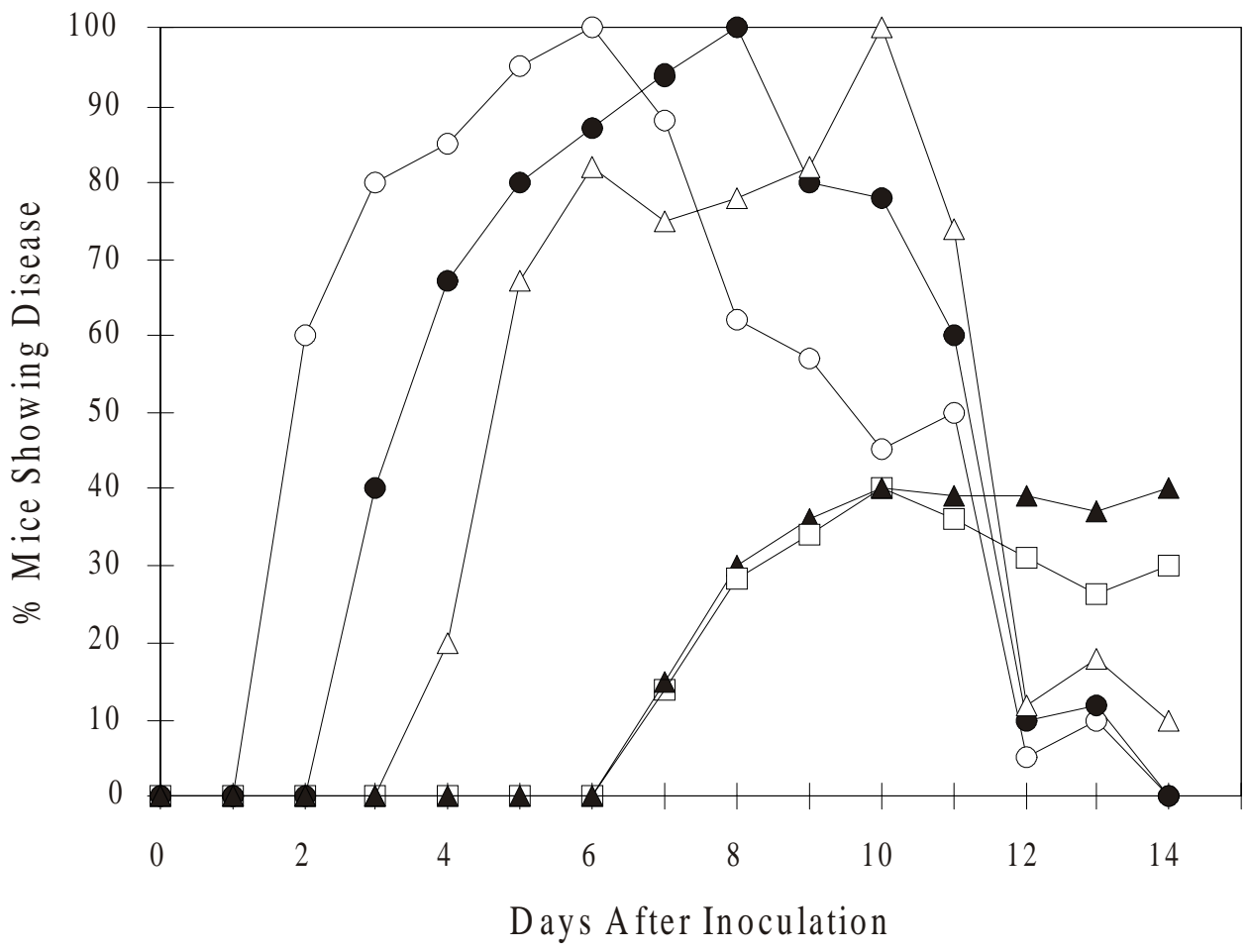


Figure 3. Graphic representation of group B mice (scarification) with HSV ophthalmic infection. The data shows that conjunctivitis $(O)$ was the first observed symptom and took 3 days to reach $100 \%$ of the group B animals. Epithelial keratitis ( $\bullet$ ) was observed since the first day a.i. reaching $100 \%$ of the animals at the $2^{\text {nd }}$ day a.i. Blepharitis $(\triangle)$ was observed between the $2^{\text {nd }}$ and $3^{\text {rd }}$ day a.i. reaching $100 \%$ of the animals at the $5^{\text {th }}$ day a.i.. Group B animals presented stromal keratitis $(\Lambda)$ and neovascularization $(\square)$ between the $3^{\text {rd }}$ and $4^{\text {th }}$ day a.i., and with a maximum of $65 \%$ and $60 \%$, respectively, of animals involvement at the $5^{\text {th }}$ day a.i.

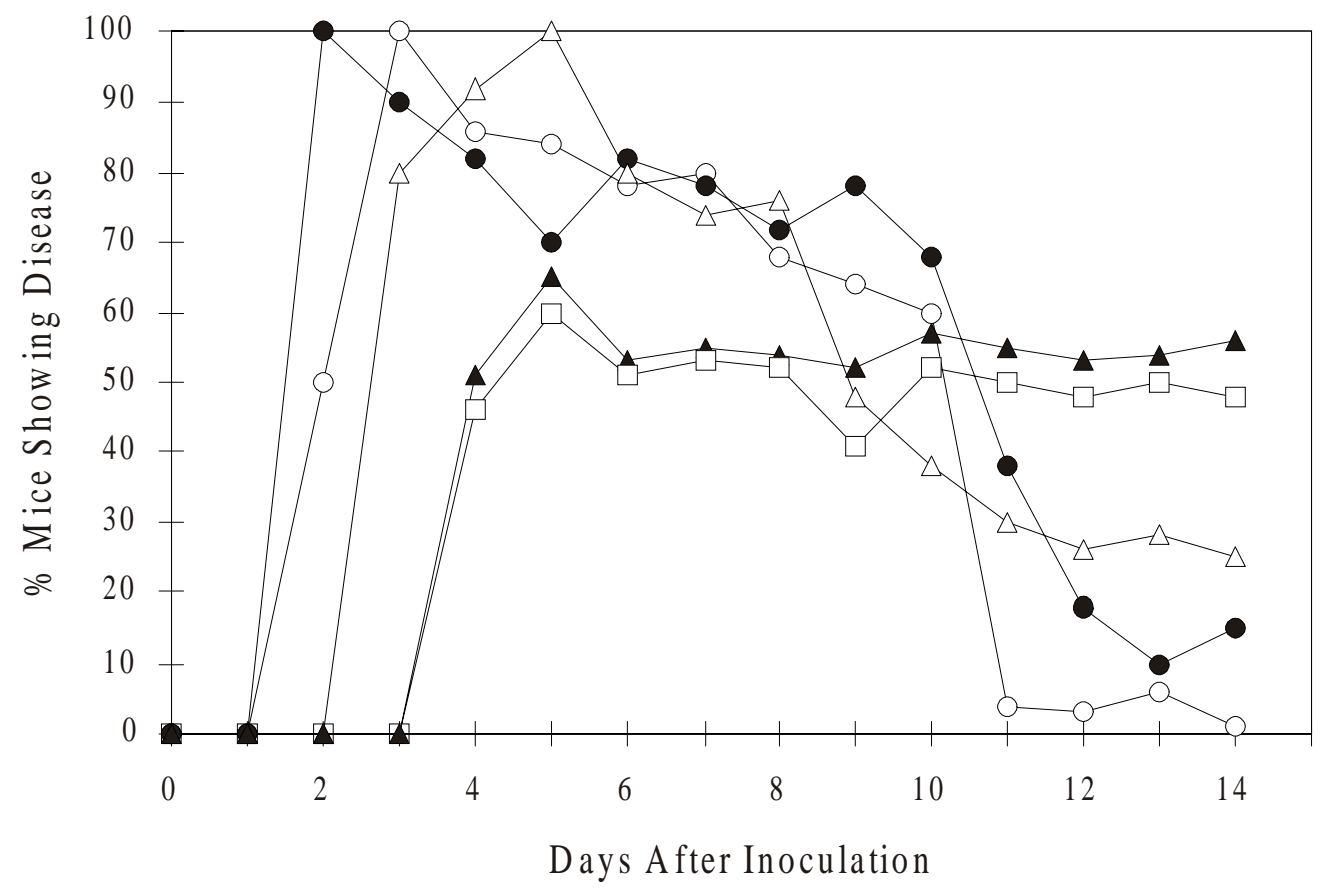


Figure 4. Mean percentage of HSV infected eyes detected by IF and by virus isolation in Vero cell culture. The cumulative percentage of mice showing ophthalmic infection at various times following ophthalmic inoculation is shown. The group A animals ( $\boldsymbol{\square}$ ) were inoculated without scarification of the cornea. The group B animals ( $\bullet$ ) were inoculated after scarification of the cornea.

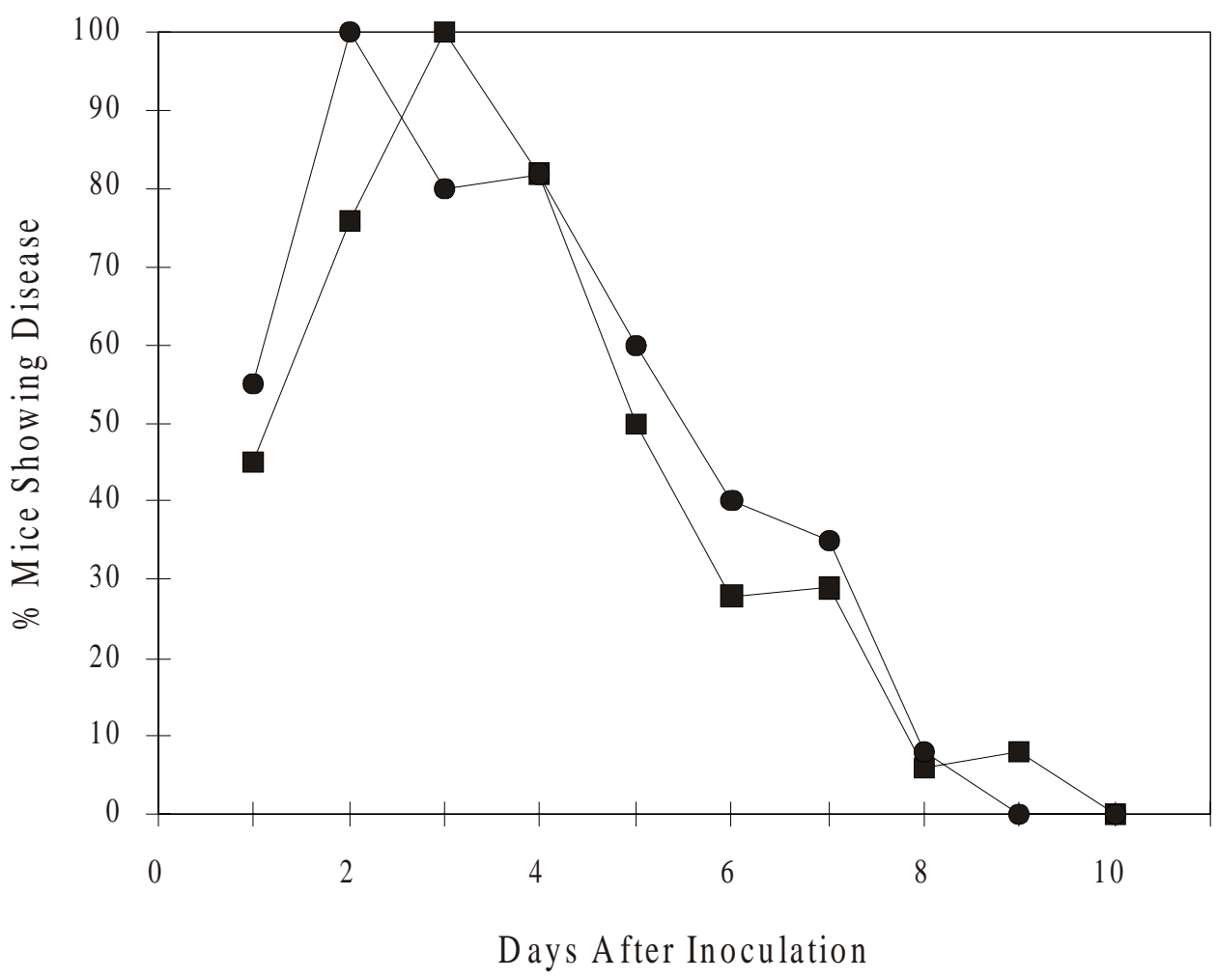


Figure 5. Cytological and histological analysis of mouse eyes by H\&E staining. A, uninfected control eye: the corneal stratified epithelium ( $\neg$ ) overlies basal membrane ( ) and Bowman's layer $(\zeta)$; in the collagenous stroma no blood vessels or lymphatics is between Bowman's layer, Descemet's membrane $(\rightarrow)$ and the endothelium ( $>$ ); under, the crystalline lens; (HE x 400). B, HSV-1 infected eye: cell in hyperplastic corneal epithelium with typical intranuclear inclusion body ( ); stromal polymorphonuclear infiltration $(\aleph)$ and proliferated mesenchymal cells $(\rightarrow$ ) in the fibrotic stroma; (HE x 1,000). C, HSV-1 infected eye: hyperplastic corneal epithelium $(\Omega)$ with degenerative cells $(\rightarrow)$; there is vascular neoformation $(>)$ in the fibrotic stroma; (HE x 400). D, HSV-1 infected eye: typical multinucleated epithelial cell ( ) in the conjunctival swab slide; (HE $\mathrm{x} 1,000)$. E, HSV-1 infected eye: corneal ulceration ( $\downarrow$ ) related to a predominantly polymorphonuclear leukocytic infiltration ( ) and stromal fibrosis $(\rightarrow)$; (HE x 250). F, HSV-1 infected eye: superficial ulceration of the eyelid conjunctiva $(\lambda)$ associated to leukocytic infiltration $(\rightarrow)$ and edema $(\boldsymbol{>})$ of the conjunctival stroma; normal Meibomius's glands ( ); (HE x 160).

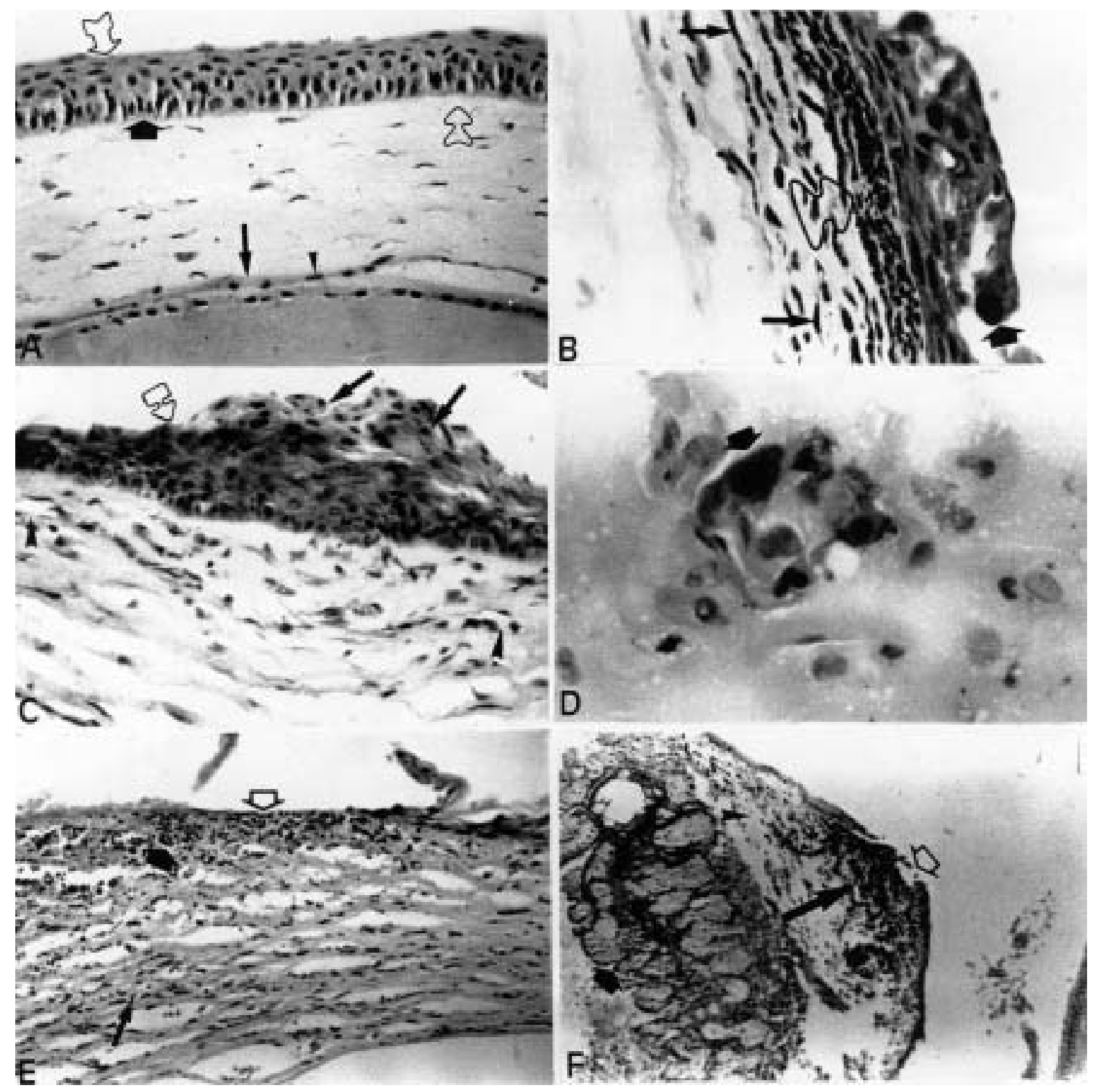


of Pathological Anatomy, IPPMG - UFRJ), Agilson Santos and João Baltazar Alves (Laboratory of Viral Pathogenesis and Diagnosis, Department of Virology, IMPPG - UFRJ) for their technical assistance.

\section{References}

1. Liesegang T.J. Epidemiology of ocular herpes simplex. Incidence in Rochester, MN, 1950-1982. Arch Ophthalmol 1989;107:1155-9.

2. Easty D.L. Clinical aspects of ocular herpes simplex virus infection. In: Viral Disease of the Eye. London: LloydLuke, 1985.

3. O’Day D.M., Jones B.R. Herpes simplex keratitis. In: Duane T.D., Jaeger E.A. eds. Clinical Ophthalmology. Cambridge: Harper \& Row, 1985.

4. Donaldson D.D. Cornea and sclera. In: Mosby C.V. ed. Atlas of external diseases of the eye. Saint Louis, 1980.

5. Tullo A.B. Herpes simplex keratitis: latent and recurrent infection. In: Easty D.L., Smolin G. eds. External eye disease. London: Butterworths, 1985.

6. Weschler S.L., Nesburn A.B. Recent advances in the molecular biology of herpes simplex latency: A short review. In: Cavanagh H.D. ed. The Cornea: Transactions of the World Congress on the Cornea. New York: Raven Press, 1988.

7. Abghari S.G., Stulting R.D. Recovery of herpes simplex virus from ocular tissue of latently infected inbred mice. Invest Ophthalmol Vis Sci 1988;29:239-43.
8. Bouley D.M., Kanangat S. Rouse B.T. The role of the innate immune system in the reconstituted SCID mouse model of herpetic stromal keratitis. Clin Immunol Immunopathol 1996;80:23-30.

9. Brandt C.R., Coakley L.M., Grau D.R. A murine model of herpes simplex virus-induced ocular disease for antiviral drug testing. J Virol Meth 1992; 36:209-22.

10. Margolis T.P., Dawson C.R., La Vail J.H. Herpes simplex viral infection of the mouse trigeminal ganglion. Invest Ophthalmol Vis Sci 1992;33:259-67.

11. Romano A., Peisich A., Wasserman D., Gamus D. Aggravation of herpetic stromal keratitis after epidermal growth factor topical application. Cornea 1994;13:167-72.

12. Schimeld C., Hill T.J., Blyth W.A., Easty D.L. Reactivation of latent infection and induction of recurrent herpetic eye disease in mice. J Gen Virol 1990;71:397-407.

13. Stulting R.D., Kindle J.C., Nahmais A.J. Patterns of herpes simplex in inbred mice. Invest Ophthalmol Vis Sci 1985;36:1360-9.

14. Valyi-Nagy T., Deshmane S., Dillner A., Fraser N.W. Induction of cellular transcription factors in trigeminal ganglia of mice by corneal scarification, herpes simplex virus type 1 infection and explanation of trigeminal ganglia. J Virol 1991;65:4142-52.

15. Maggs D.J., Chang E., Nasisse M.P., Mitchell W.L. Persistence of herpes simplex virus type 1 DNA in chronic conjunctival and eyelid lesions of mice. J Virol 1998;72:9166-72.

16. Yan X.T., Tumpey T.M., Kunkel S.L., et al. Role of MIP-2 in neutrophil migration and tissue injury in the herpes simplex virus 1 infected cornea. Invest Ophthalmol Vis Sci 1998;39:1854-62. 